\title{
Reference Leaf Transcriptomes for Potato Cultivars: Desiree and PW363
}

Maja Zagorščak ${ }^{1}$, Marko Petek $^{1}$, Mohamed Zouine ${ }^{2}$, Živa Ramšak $^{1}$, Kristina Gruden ${ }^{1}$

National Institute of Biology - Department of Biotechnology and Systems Biology, Ljubljana, Slovenia

${ }^{2}$ Ecole Nationale Supérieure Agronomique de Toulouse, Toulouse, France

Web: http://www.nib.si, Email: Maja.Zagorscak@nib.si, Phone: +386 (0)59232 843

\section{Objectives}

Assemble reference leaf transcriptomes for Desiree and PW363 cultivars to assist with:

- StNIB-v1 potato pangenome expansion with novel genes and allels

- improved quantification with RNASeq in further experiments

\section{Introduction}

Most potato cultivars are highly heterozygous tetraploids with high genetic variability.

To bypass polyploidy related sequencing problems, Potato Genome Sequencing Consortium (PGSC, 2011) sequenced a double monoploid derived from S. tuberosum group Phureja. While this approach resulted in a acceptable gene model, it does not cover all genes and gene variants present in cultivated potato.

In order to avoid this problems, to discriminate between paralogous genes and to identify traits that are not present in initially sequenced genotype, RNA-sequencing for cv. Desiree and cv. PW363 leaves was conducted on Illumina NGS platform.

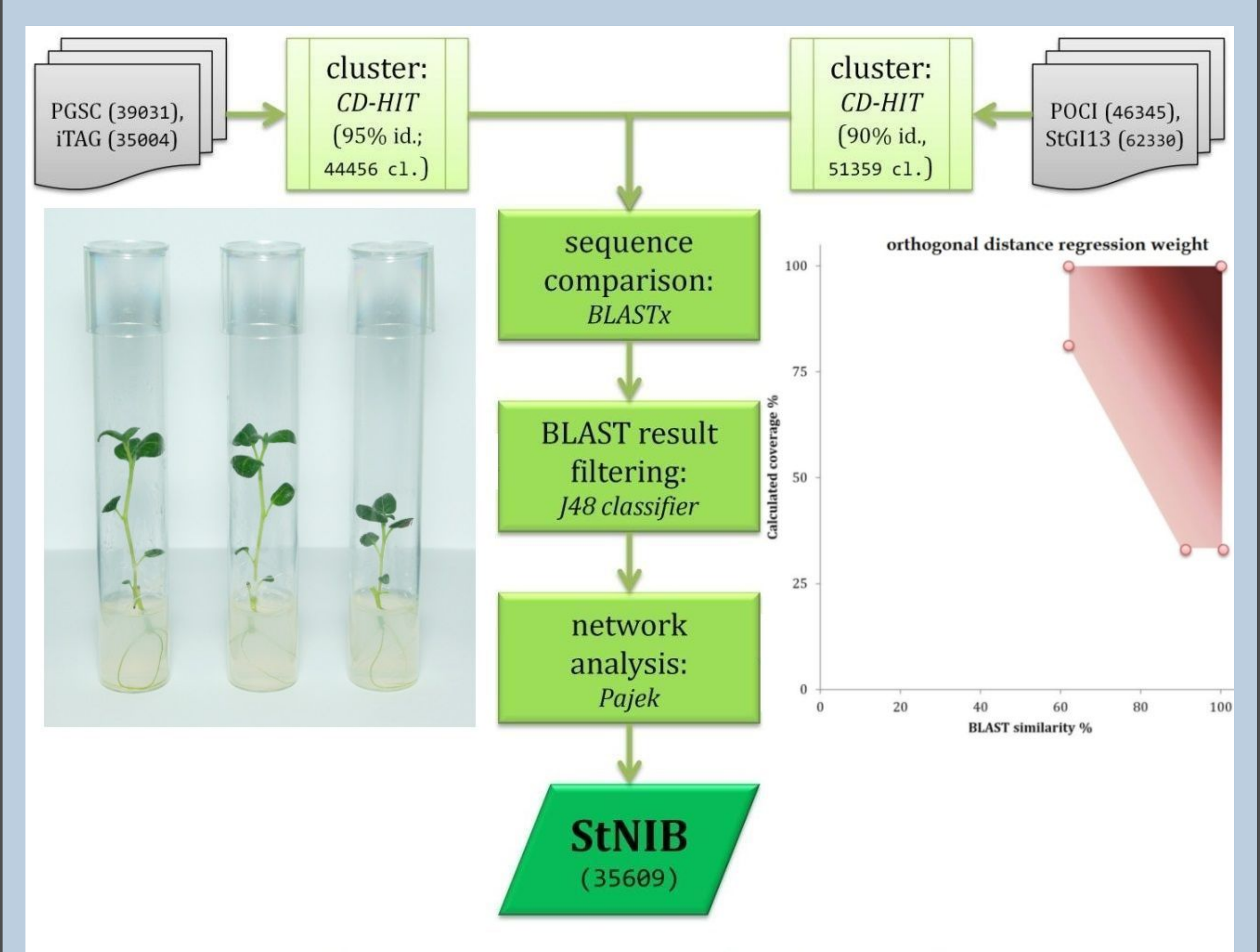

StNIB-v1 potato pangenome

\section{Conclusion}

Initial potato pangenome containing 35609 genes was expanded with 24999 potential new transcripts, and will serve to further expand knowledge on the potato pathogen interactions.

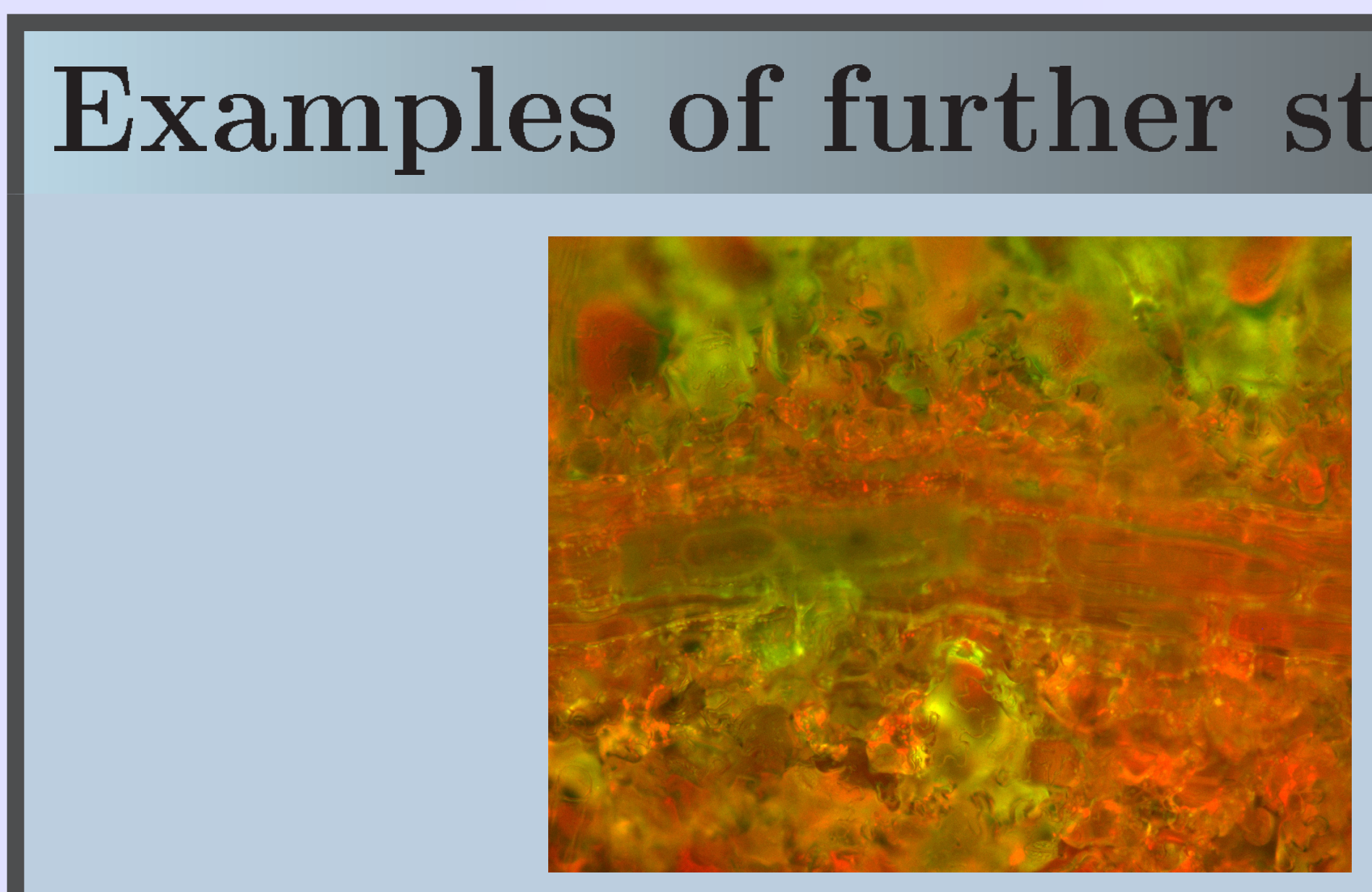

Confocal microscopy

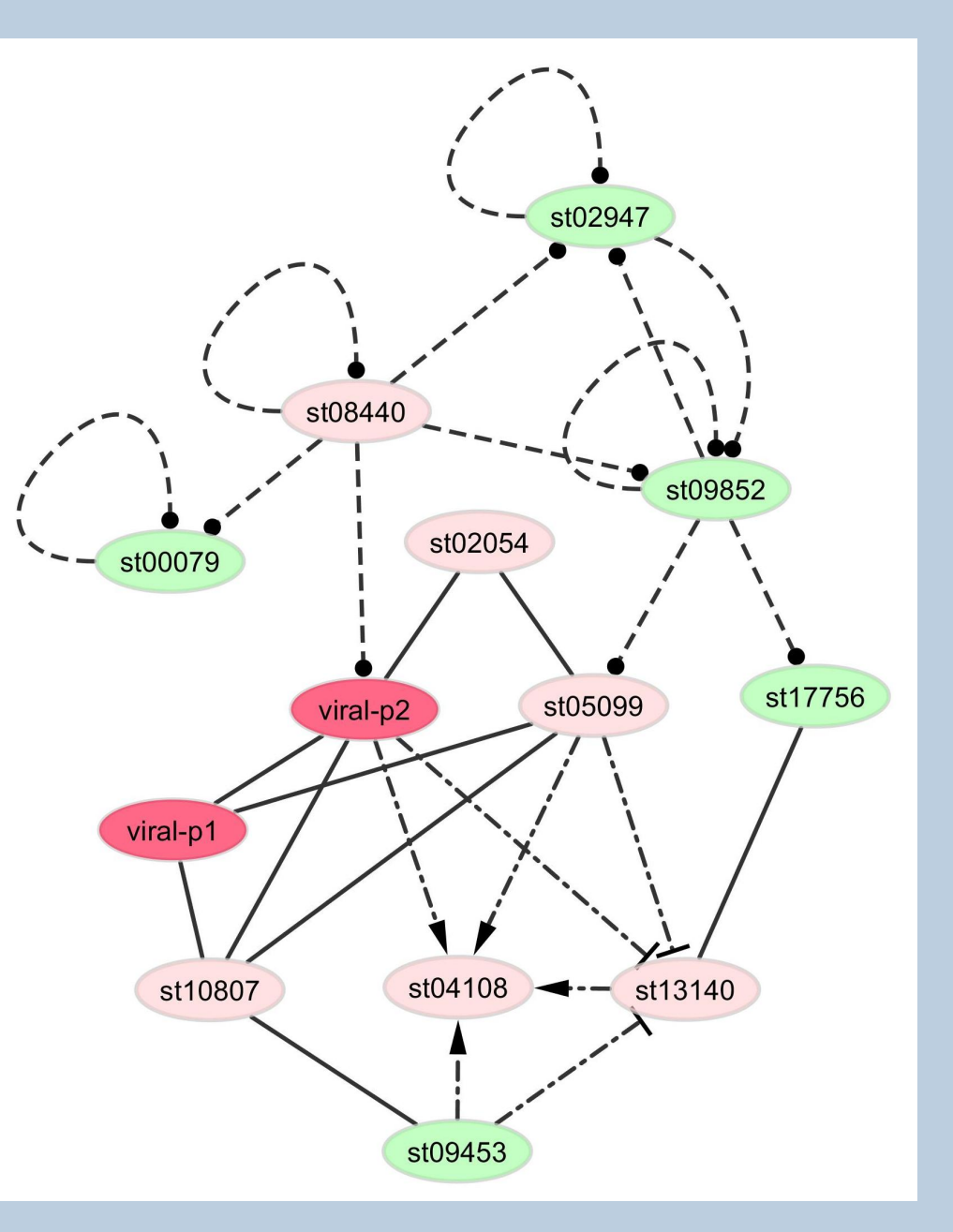

Network analysis

\section{Workflow and results}

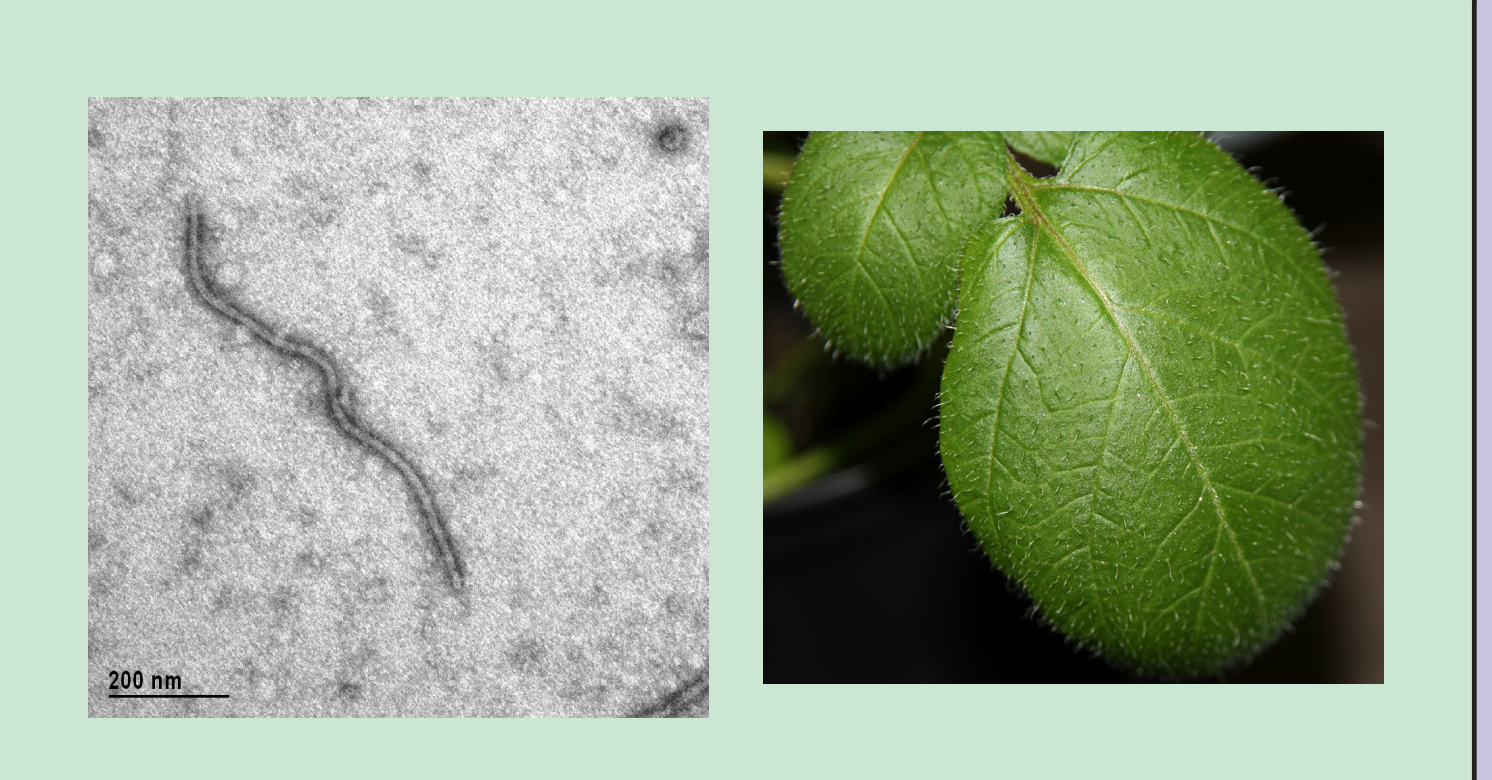
complemented with deposited dat Desiree: 26949735 raw reads PW: 52171015 raw reads

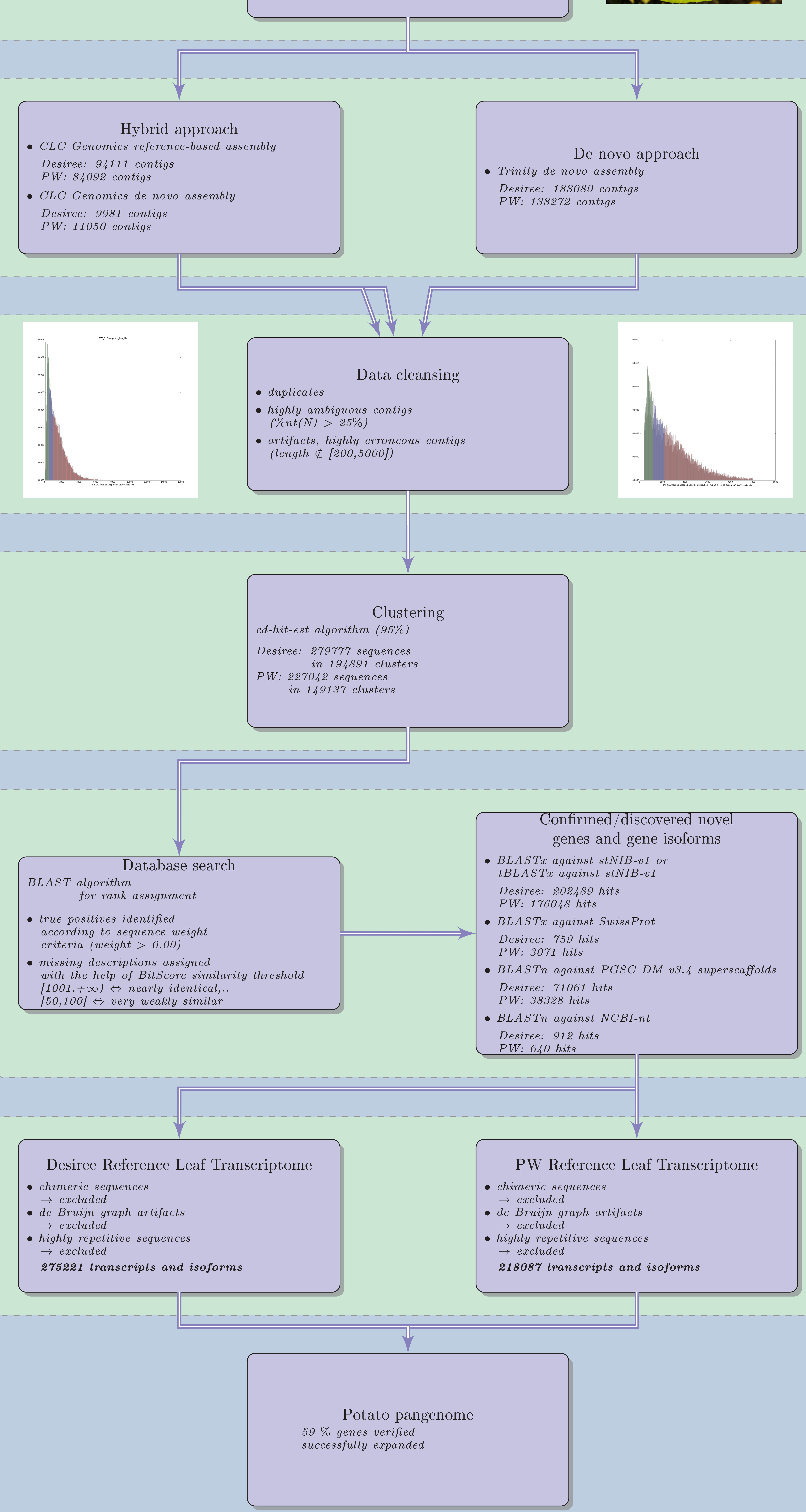

